\title{
Screening of factors that influence the preparation of Dialium guineense pods active carbon for use in methylene blue adsorption: a full factorial experimental design
}

\author{
Enebi Estella Jasper ${ }^{{ }^{*}} \mathbb{0}$, Jude Chinedu Onwuka ${ }^{2}$ and Yakubu Manasseh Bidam ${ }^{1}$
}

\begin{abstract}
Background: The use of active carbons derived from waste biomass as adsorbents in the remediation of wastewater remains a valuable and cost-effective technology when compared to the use of commercial active carbon for the same purpose. This research aims at using a 2-level full factorial design (FFD) to efficiently evaluate factors that influence the preparation of active carbon from the waste pods of the Dialium guineense seeds. The influence of three preparation factors (concentration of the activating agent, activation time, and type of activating agent) on the active carbon yield and its adsorption capacity for methylene blue were investigated. Based on the full factorial design, two regression models were developed to correlate the factors to the two responses. From an analysis of variance (ANOVA), the most significant factors influencing each response were identified. The active carbon preparation conditions were then optimized by maximizing both the active carbon yield and its adsorption capacity for Methylene Blue. The functional group and surface morphology of the active carbon prepared under the predicted optimum conditions was analyzed via Fourier Transform Infra-Red (FTIR) spectroscopy and Scanning Electron Microscopy (SEM) respectively.

Results: The results of this study revealed that the concentration of the activating agent had the most significant effect on the yield of the active carbon produced as well as on its adsorption capacity for methylene blue. The optimum preparation process conditions were found to be: concentration of activating agent $5 \mathrm{M}$, activation time 30 min and activating agent, $\mathrm{NaOH}$ which resulted in an active carbon yield of $21.25 \%$, and an adsorption capacity of 9.33509 mg MB per gram of active carbon. SEM and FTIR showed evidence of successful activation.

Conclusion: The preparation of Dialium guineense seed pods' active carbon is strongly influenced by concentration and type of activating agent used. Reliable statistical models based on the FFD proved to be useful in identifying factors that significantly influence the preparation of Dialium guineense seed pods' active carbon. The active carbon holds great potential for application in the elimination of hazardous synthetic dyes from wastewater and should be explored further.
\end{abstract}

Keywords: Active carbon, Screening, Optimization, Full factorial design, Dialium guineense

${ }^{*}$ Correspondence: enebijasper@yahoo.com

${ }^{1}$ Department of Applied Chemistry, Kaduna Polytechnic, Kaduna, Kaduna State, Nigeria

Full list of author information is available at the end of the article

\section{Background}

Water pollution precipitated by dye-using industries is a source of global environmental concern especially as most dyes are known toxicants (probable carcinogens or mutagens) to both human and aquatic lives (Yagub 
et al. 2014). Methylene blue (MB) is a synthetic thiazine dye used extensively in several industries. It is a dye most commonly found in wastewater discharged from diagnostic laboratories and textile industries. Methylene blue, when exposed to humans, has been reported to cause increased heart rate (tachycardia), headache, nausea, convulsions, and gastritis (Gouamid et al. 2013). It is also a known toxicant to neonates and the central nervous system (Albert et al. 2003; Gillman 2011). Hence, its removal from water remains pertinent.

Several treatment technologies have been employed to sequester dyes as pollutants from aqueous solutions. Adsorption has been described as one of the most effective methods to remove such dyes from contaminated waters owing to its simplicity and cost-effectiveness (El Haddad et al. 2013; Kyzas et al. 2013). The use of active carbon as an adsorbent is highly recommended, however, the high cost of commercial active carbon limits its efficiency as an adsorbent especially for application in developing countries. This high cost has prompted researchers to explore the use of agro-waste-derived active carbon as a low-cost adsorbent. The conversion of agricultural waste materials into value-added products such as active carbon is a viable technology that solves the pollution problem and reduces the cost of wastewater treatment as commercial active carbon is usually very expensive and out of reach of low and small-scale industries. Over the years, the demand for active carbon has increased considerably as a water-treatment agent to mitigate the environmental risks associated with water pollution. In recent years, the preparation of active carbon from cheap agricultural wastes, such as corn cob (Campos et al. 2020), rice husks (Menya et al. 2020), groundnut shell (Kumari et al. 2020), durian shell (Wang et al. 2020), walnut shells (Bae et al. 2014), and so on, has drawn extensive attention from researchers. Some of these waste materials are potential precursors that could be employed to make valuable products, such as adsorbents.

The preparation of active carbon is influenced by many factors, some of which include; activation temperature, type of activating agent, and concentration of the activating agent, activating temperature, etc. The study of the effects of such factors using the traditional "one-factorat-a-time" (OFAT) approach to experimentation, where one factor is optimized while others remain constant is time-consuming and somewhat tedious. The use of factorial experimental design can minimize the aforementioned difficulties by optimizing all the affecting parameters simultaneously (Montgomery 2013). Factorial experimental designs involve applying mathematical and statistical techniques to decrease the total number of experiments required to achieve the best optimization of a process. The effect of each factor on a response, as well as how the effect of each factor varies with the change in the level of the other factors are determined by the design. The main advantages of this experimental design are; the effects of individual parameters or factors as well as their relative importance are obtained, and the interaction between two or more factors can be established (Geyikçi and Büyükgüngör 2013). This paper aims to demonstrate the use of the seed pods of Dialium guineense, an agro-derived waste, for the preparation of active carbon and to evaluate the influence of selected preparation factors on its performance in terms of its yield and adsorptive capacity for methylene blue. To date, there are limited studies concerning the application of this factorial experimental design in the production of active carbon from Dialium guineense seed pods.

\section{Methods}

Dialium guineense seed pods (DGP) were obtained from Bakin Dogo, Kaduna State, Nigeria and authenticated at the Herbarium of the Department of Botany at the Ahmadu Bello University, Zaria, with voucher number ABU0243. The pods were deseeded, washed with tap water, and then with deionized water to remove dirt from its surface, and subsequently dried at $100{ }^{\circ} \mathrm{C}$ until a constant weight was achieved. The dried precursors were crushed and screened to $2-4 \mathrm{~mm}$ using standard sieves and preserved in clean containers.

The adsorbate, Methylene blue dye was used without purification. All reagents used were of analytical grade. Standard solutions were prepared with deionized water and the $\mathrm{pH}$ of the dye solutions adjusted to $\mathrm{pH} 7$ for all experiments.

\section{Factorial experimental design for the preparation of DGP active carbon}

Factorial design experiments permit the simultaneous study of the effects that several factors have on a particular process. They also allow interactions between different groups of factors to be measured. A common factorial experimental design is one with all input factors set at two levels, usually designated as 'high' and 'low' or ' +1 ' and ' -1 '. If there are $n$ factors each at two levels, a full factorial design has $2^{n}$ runs. In the present study, a three-factor two-level full factorial design ( $2^{3}$ runs) was used for modeling the active carbon preparation process. The factors (type of activating agent, concentration of activating agent, activation time) were varied at two levels as given in Table 1 .

The total number of experiments for the production of DGP active carbon as determined by the statistical software (Minitab 17.0) through the design of experiment (DOE) was 10 comprising of 8 runs with two center points. Chemical activation involving the impregnation 
Table 1 Levels of factors applied for the preparation of DGP active carbon

\begin{tabular}{lll}
\hline Factor & Low level $(\mathbf{- 1 )}$ & High level $(+\mathbf{1})$ \\
\hline Activating agent] (M) & 1 & 5 \\
Activation time (min) & 30 & 90 \\
Activation agent type & $\mathrm{H}_{3} \mathrm{PO}_{4}$ & $\mathrm{NaOH}$ \\
\hline
\end{tabular}

with activated agents, $\mathrm{H}_{3} \mathrm{PO}_{4}$ and $\mathrm{NaOH}$ followed by thermal treatment (carbonization) at $500{ }^{\circ} \mathrm{C}$ at different times was adopted to produce the active carbons.

Experiments for the Full Factorial Design (FFD) for the preparation of the active carbons were carried out as follows: $10 \mathrm{ml}$ of the desired concentration of the activating agent $\left(\mathrm{NaOH}\right.$ and $\left.\mathrm{H}_{3} \mathrm{PO}_{4}\right)$ was mixed separately with $5 \mathrm{~g}$ of raw Dialium guineense in $30 \mathrm{ml}$ porcelain crucibles. The mixtures were left to soak for $30 \mathrm{~min}$ at room temperature for increased percolation. The impregnated samples were dehydrated for $1 \mathrm{~h}$ at $110{ }^{\circ} \mathrm{C}$ after which they were transferred to a muffle furnace set at a constant temperature of $500{ }^{\circ} \mathrm{C}$ at activation times as depicted in Table 2. At the end of the pyrolysis, the sample active carbons were gradually cooled in a desiccator and then rinsed thoroughly with deionized water until neutral $\mathrm{pH}$ was achieved. The active carbons were dried for $1 \mathrm{~h}$ at $110^{\circ} \mathrm{C}$ and stored in sealed glass bottles until further use. The performance of the active carbon preparation process was evaluated by determining the percentage yield of the active carbons produced and their adsorption capacities for MB.

\section{Percent yield}

The active carbon yield was calculated as the ratio of the weight of dried active carbon to the initial weight of the precursor (DGP), both based on a dry basis and expressed as a percentage. The percentage yield and adsorption capacity for methylene blue of the active carbons produced were used to optimize the preparation process.

\section{Adsorption experiments}

Batch adsorption experiments were carried out by agitating $0.1 \mathrm{~g}$ of each prepared activated carbon with $50 \mathrm{ml}$ of $25 \mathrm{mg} / \mathrm{l}$ of $\mathrm{MB}$ solution in stoppered $250 \mathrm{ml}$ conical flasks for $60 \mathrm{~min}$ at ambient temperature after which the suspensions were centrifuged and dye concentrations in the supernatant solutions were measured. Standard curves were developed at $\lambda \max$ of $664 \mathrm{~nm}$ for methylene blue dye through the measurement of the dye solution absorbance by UV/Visible Spectrophotometer (Agilent CARY 300). The amount of dye adsorbed per unit mass of adsorbent was calculated using the following equation:

$$
q_{e}=\frac{\left(C_{o}-C_{\mathrm{e}}\right) V}{m}
$$

$q$ where $q_{\mathrm{e}}$ is the amount of dye adsorbed on the adsorbent $(\mathrm{mg} / \mathrm{g}), C_{0}$ and $C_{\mathrm{e}}(\mathrm{mg} / \mathrm{L})$ are the liquid-phase concentrations of the dye at initial and equilibrium, respectively. $V(\mathrm{~L})$ is the volume of dye solution and $(\mathrm{g})$ is the amount of the active carbon used.

\section{Statistical evaluation of the effect of the factors on the active carbon preparation process}

The results of the experimental design were analyzed using Minitab 17 statistical software (Minitab Inc., USA) to evaluate the effect of the factors on the preparation process as well as their statistical significance. This was achieved by the use of multiple regression analysis, analysis of variance (ANOVA), and the use of the statistical plots (Pareto plots and Normal probability plots of the standardized effects). Multiple regression analysis

Table 2 Experimental design matrix and responses for the preparation of Dialium Guineense pods activated carbon (DGPAC)

\begin{tabular}{|c|c|c|c|c|c|}
\hline Run order & [Activating agent] $\mathrm{M}$ & Activation time (min) & Activating agent & Yield (\%) & $\begin{array}{l}\text { Adsorption } \\
\text { capacity } \\
(\mathrm{mg} / \mathrm{g})\end{array}$ \\
\hline 1 & 1 & 90 & $\mathrm{NaOH}$ & 32.40 & 4.607 \\
\hline 2 & 5 & 90 & $\mathrm{H}_{3} \mathrm{PO}_{4}$ & 23.45 & 5.513 \\
\hline 3 & 5 & 30 & $\mathrm{NaOH}$ & 26.00 & 9.628 \\
\hline 4 & 5 & 30 & $\mathrm{H}_{3} \mathrm{PO}_{4}$ & 27.99 & 7.003 \\
\hline 5 & 3 & 60 & $\mathrm{NaOH}$ & 27.26 & 4.550 \\
\hline 6 & 1 & 30 & $\mathrm{NaOH}$ & 41.24 & 2.920 \\
\hline 7 & 1 & 90 & $\mathrm{H}_{3} \mathrm{PO}_{4}$ & 32.74 & 2.700 \\
\hline 8 & 5 & 90 & $\mathrm{NaOH}$ & 25.14 & 9.534 \\
\hline 9 & 1 & 30 & $\mathrm{H}_{3} \mathrm{PO}_{4}$ & 44.58 & 2.365 \\
\hline 10 & 3 & 60 & $\mathrm{H}_{3} \mathrm{PO}_{4}$ & 30.72 & 2.090 \\
\hline
\end{tabular}


applied to the experimental data generated regression model equations that depict the empirical relationships between the responses (i.e. activated carbon yield $\left(Y_{1}\right)$ and adsorption capacity of $\mathrm{MB}\left(Y_{2}\right)$, and the three independent variables or factors (concentration of activating agent $(A)$, activating time $(B)$ and activating agent $(C)$. The factors that significantly influence the preparation process and their optimum levels were determined by using the ANOVA which includes Fisher's $F$-test (for overall model significance), its associated probability $p(F)$, and coefficient of determination, $R^{2}$.

\section{Characterization of DGP and DGPAC produced under optimal conditions}

The activated carbon produced under optimal conditions and its precursor were characterized using scanning electron microscopy and Fourier transform infra-red spectroscopy.

\section{Scanning electron microscopy}

The scanning electron microscopy (SEM) technique was employed to observe the surface morphology of the DGPAC and its precursor. All measurements were carried out using the Phenom ProX 800-07334 Scanning Electron Microscope (Eindhoven, Netherlands) within an accelerated voltage of $10 \mathrm{kV}$ in a vacuum condition.

\section{Fourier transform infra-red spectroscopy}

FTIR spectra for the precursor (DGP) and active carbon prepared under optimum conditions were qualitatively identified using the FTIR CARY 630 (Agilent Technologies) within the range of $4000-650 \mathrm{~cm}^{-1}$.

\section{Results}

Preparation of DGPAC via the factorial experimental design Table 2 represents the complete experimental design matrix with all the responses obtained from the experiments for the production of DGPAC. The values of activated carbon yield ranged from 23.45 to $44.58 \%$ while the adsorption capacity for MB ranged from 2.090 to $9.638 \mathrm{mg} / \mathrm{g}$.

\section{Statistical assessment of the influence of preparation factors on the responses Regression equations}

For each response, model regression equations were derived after which each model was reduced by removing insignificant terms. The regression Eqs. (2) and (3) describe the empirical relationship between the factors (concentration of activating agent, activation time, and activation agent) and the responses (active carbon yield, and adsorption capacity for $\mathrm{MB}$ ). The magnitude of the coefficients in the equations shows the degree of influence a term or factor has on the response variable, while the sign of the coefficient depicts the direction of the influence the factor has on a response variable. The positive sign of the coefficients in a regression equation indicates a synergistic or positive effect, while the negative sign represents a negative or antagonistic effect on the response (Kalavathy et al. 2009).

$$
\begin{aligned}
& Y_{1}(\text { activecarbonyield }) \\
& \quad=31.152-6.048 A-3.260 B+1.910 A B \\
& Y_{2} \text { (Adsorptioncapacity) } \\
& =5.5337+2.3858 A+0.0549 B+1.1567 C \\
& \quad-0.4507 A B+0.5229 A C+0.3435 B C
\end{aligned}
$$

\section{Analysis of variance (ANOVA)}

The suitability of the models is justified through an analysis of variance. ANOVA defines the statistical significance of the models and describes the effects of each process factor and the interactions between them on the response (Das and Mishra, 2017). The ANOVA for the two quadratic models is presented in Table 3.

The ANOVA describes the precision of the actual relationship between the response and significant factors as represented by the model equation. Fisher's $F$-test $(F)$ and Prob $>F(p)$ values which are included in the ANOVA connote the significance of a process factor. A larger $F$ and a smaller $p$, indicate that a factor is more significant (Reddy et al. 2015). " $p$ "-values less than 0.05, suggest that the influence of the corresponding factor on the response is statistically significant. Coefficients of determination $\left(R^{2}\right)$ values were also used to evaluate the fitness of the model equations in predicting the experimental responses. An $R^{2}$ value closer to $100 \%$ confirms the fitness of the model. In other words, the more the $R^{2}$ value approaches $100 \%$, the higher the degree of correlation between the experimental and predicted values, hence the higher the accuracy of the obtained model.

\section{Pareto and normal plots}

Pareto plots and normal plots are diagnostic plots that show a graphical representation of the magnitude and the effect of factors on the responses. The Pareto plot is an ordered bar chart of the normalized coefficients, which represent the relative importance of the individual and the interaction effects of factors on the response. The Pareto chart displays an absolute value of each effect and draws a vertical reference line on the chart which represents the magnitude of the minimum statistically significant effect at the $95 \%$ confidence level. Any factor term that extends beyond this reference line has a statistically 
Table 3 ANOVA for the yield and adsorption capacity of DGPAC

\begin{tabular}{|c|c|c|c|c|c|}
\hline Source & DF & Adj SS & Adj MS & $F$-value & $P$-value \\
\hline \multicolumn{6}{|l|}{ Yield of DGPAC } \\
\hline Model & 3 & 406.78 & 135.595 & 30.45 & 0.001 \\
\hline A: [Activating agent] & 1 & 292.58 & 292.578 & 65.71 & 0.000 \\
\hline B: Activation time (min) & 1 & 85.02 & 85.021 & 19.09 & 0.005 \\
\hline$A B$ & 1 & 29.18 & 29.185 & 6.55 & 0.043 \\
\hline Curvature & 1 & 11.69 & 11.686 & 3.89 & 0.106 \\
\hline Error & 6 & 26.72 & 4.453 & & \\
\hline Total & 9 & 433.50 & & & \\
\hline \multicolumn{6}{|c|}{ S: $2.11010, R^{2}: 93.84 \%, R^{2}$ (adj): $90.76 \%, R^{2}$ (pred): $86.11 \%$} \\
\hline Source & DF & Adj SS & Adj MS & $F$-value & $P$-value \\
\hline \multicolumn{6}{|c|}{ Adsorption capacity of DGPAC for methylene blue } \\
\hline Model & 7 & 71.5369 & 10.2196 & 1493.55 & 0.001 \\
\hline A: [Activating agent] & 1 & 45.5371 & 45.5371 & 6655.07 & $<0.001$ \\
\hline B: Activation time (min) & 1 & 0.0241 & 0.0241 & 3.52 & 0.202 \\
\hline C: Activating agent & 1 & 13.3789 & 13.3789 & 1955.27 & 0.001 \\
\hline$A B$ & 1 & 1.6247 & 1.6247 & 237.44 & 0.004 \\
\hline$A C$ & 1 & 2.1878 & 2.1878 & 319.74 & 0.003 \\
\hline$B C$ & 1 & 0.9438 & 0.9438 & 137.93 & 0.007 \\
\hline Curvature & 1 & 7.8406 & 7.8406 & 1145.87 & 0.001 \\
\hline Error & 2 & 0.0137 & 0.0068 & & \\
\hline Total & 9 & 71.5506 & & & \\
\hline \multicolumn{6}{|c|}{ S: $0.0827193, R^{2}: 99.98 \%, R^{2}(\mathrm{adj}): 99.91 \%, R^{2}$ (pred): $99.72 \%$} \\
\hline
\end{tabular}

DF, degrees of freedom; Adj.SS, adjusted sum of squares; Adj MS, adjusted mean of squares; $F$-value, Fisher's test value; $P$-value, probability value

significant effect on the response (Bingol et al. 2010). The normal plots on the other hand are capable of showing the magnitude and direction of the effects of the factors on the response. The Pareto and normal plots for DGPAC yield and MB adsorption capacity are depicted in Figs. 1 and 2 respectively.
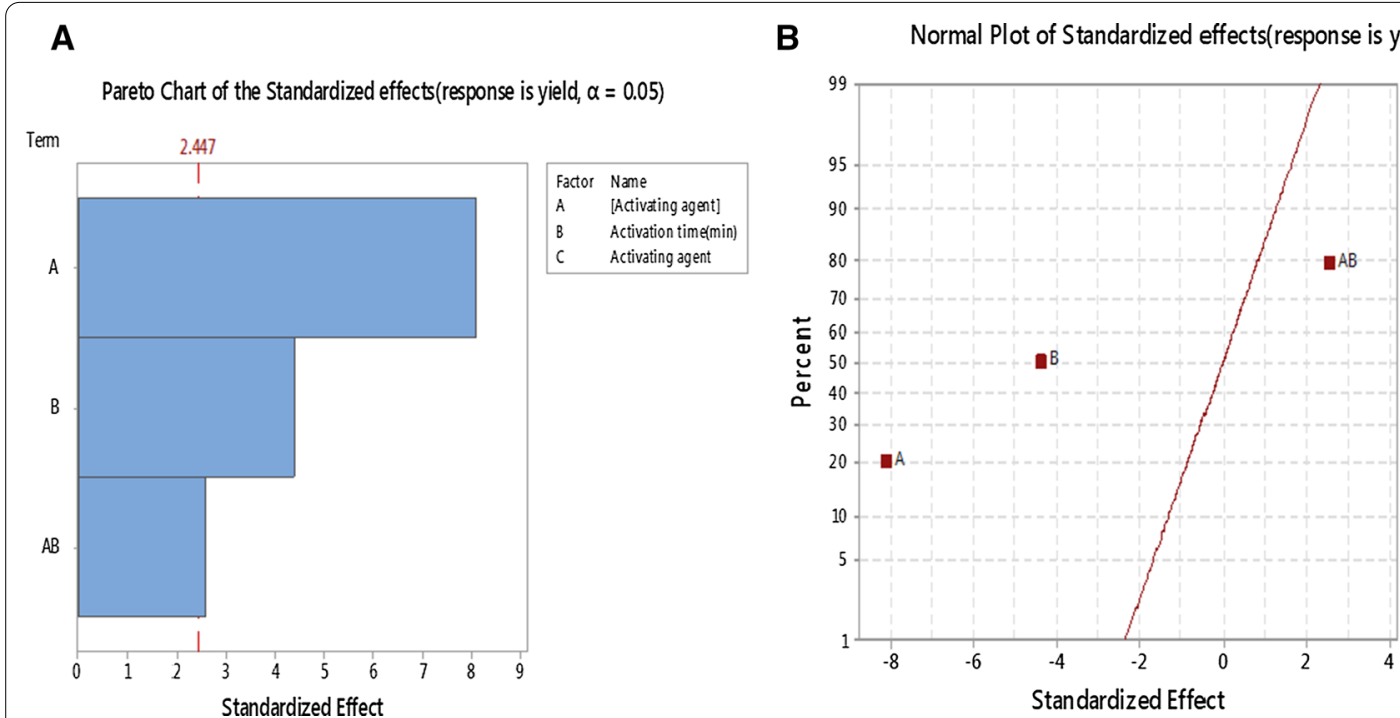

Fig. 1 a, b Pareto plot and normal plot of standardized effects of model parameters on the DGPAC yield 


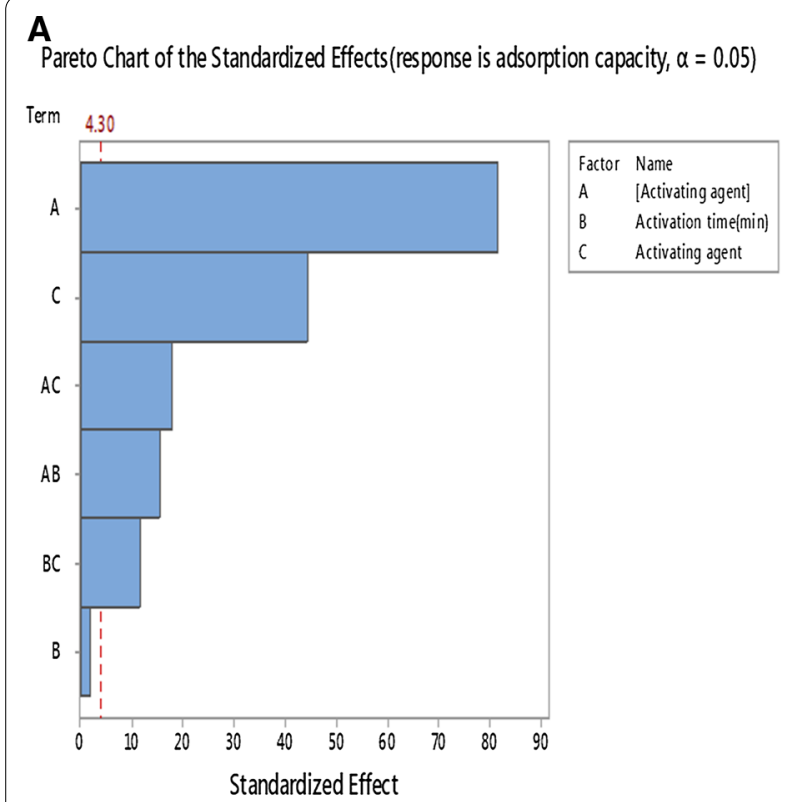

B Normal Plots of the Standardized effects (response is adsorption capacity, $\alpha=0.05$ )

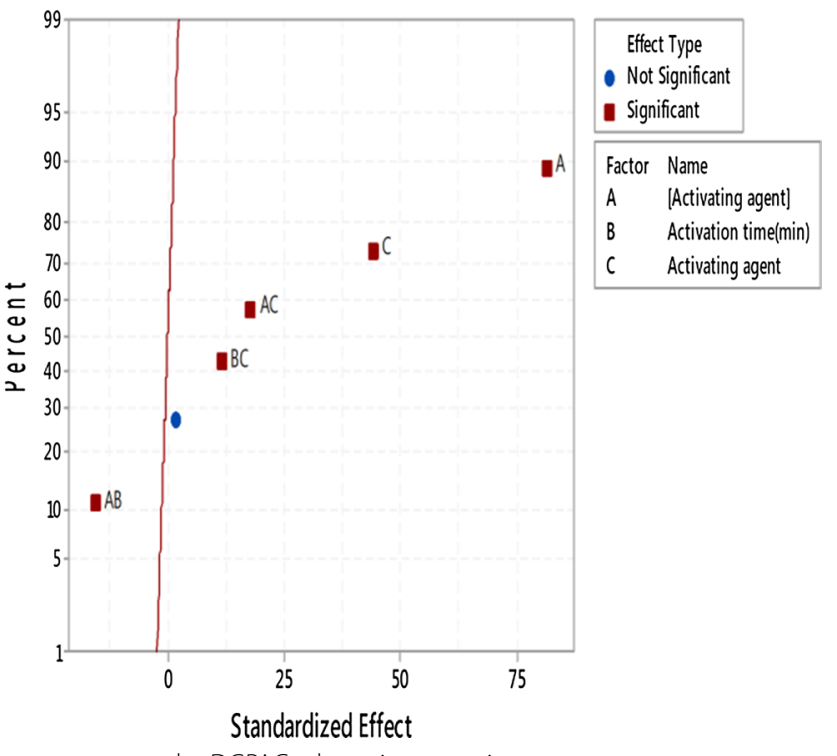

Fig. 2 a, b Pareto plots and normal plot of standardized effects of model parameters on the DGPAC adsorption capacity

\section{Process optimization and validation}

For economic feasibility in commercial production, a high product yield and a high adsorption capacity should characterize the active carbon. However, this is difficult to achieve for both responses under the same conditions, since the regions of interest of each factor are somewhat different. For instance, at a higher concentration of activating agent, a lower yield is expected but with the associated increasing development of pores, a higher adsorption capacity results. Hence as yield increases, adsorption capacity will decrease and vice versa. In an attempt to determine an acceptable compromise between all responses simultaneously, to achieve optimal factor settings that will maximize both the yield and the adsorption capacity of the active carbon, the desirability function approach as described by Derringer and Suich (1980) was applied by the software where all the response values were all set to maximum. The predicted optimal preparation process conditions obtained within the range studied were presented in Table 4 as follows: concentration of activating agent $5 \mathrm{M}$, activation time 30 min, and type activating agent $\mathrm{NaOH}$ (Table 4).

Validation experiments were carried out to verify the results from the analysis. The average activated carbon yield of $21.255 \%$ and adsorption capacity of $9.335 \mathrm{mg} \mathrm{MB}$ per gram of activated carbon were obtained (Table 4).

\section{Surface and functional group analysis of DGP and DGPAC FTIR spectra}

FTIR spectroscopy provides basic spectral information about activated carbons especially for the determination of types and intensities of their surface functional groups (El-Hendawy 2006). Figure 3 reveals major changes in the spectrum of DGPAC when compared to that of its precursor (DGP). The FTIR spectrum of raw Dialium guineense seed pods spectrum contains some distinct peaks at $3308.9 \mathrm{~cm}^{-1}$ (characteristic absorption peak of $\mathrm{O}-\mathrm{H}$ and $\mathrm{N}-\mathrm{H}$ symmetric stretching vibration), $2922.2 \mathrm{~cm}^{-1}$ (carboxylic acid O-H), $2851.4 \mathrm{~cm}^{-1}$ (symmetric $\mathrm{C}-\mathrm{H}$ stretching vibration from $\mathrm{CH}_{2}$ and $\mathrm{CH}_{3}$ ), $1722.0 \mathrm{~cm}^{-1}$ (C-O asymmetric stretching vibration) and $1021.3 \mathrm{~cm}^{-1}$ ( $\mathrm{S}=\mathrm{O}$ or $\mathrm{C}-\mathrm{O}$ stretching). Most of these distinct were absent in the spectra of the DGPAC.

\section{SEM analysis of DGP and DGPAC}

Figure $4 \mathrm{a}, \mathrm{b}$ show the SEM micrographs which depict the surface morphology of the raw Dialium guineense seed pods (DGP) and the optimal Dialium guineense seed pods activated carbon (DGPAC) respectively.

\section{Discussion}

Evaluation of the influence of the preparation factors on the responses Interpretation of regression models and ANOVA

The response, active carbon yield, is described by the model equation Eq. (2), which shows that the activating agent concentration $(A)$ and activation time $(B)$ had 


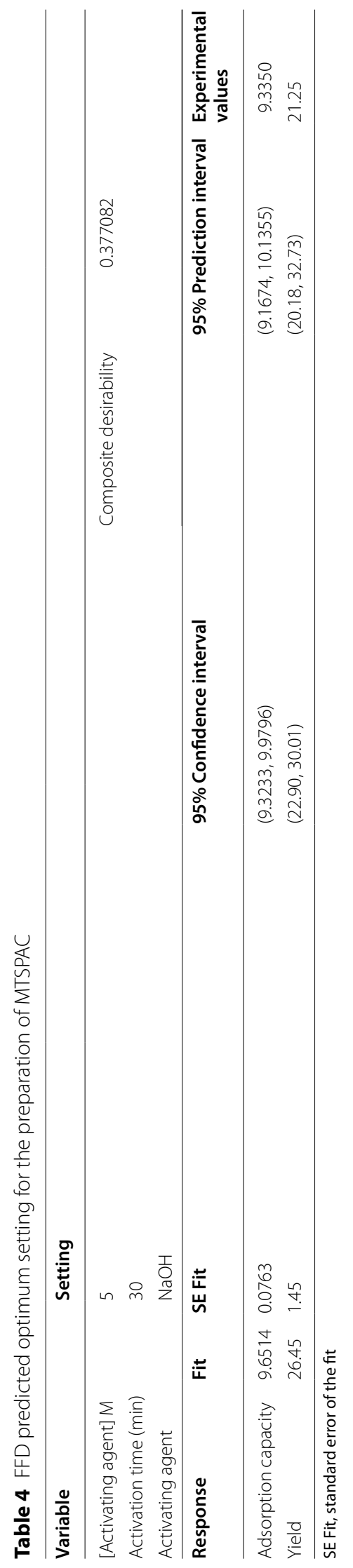




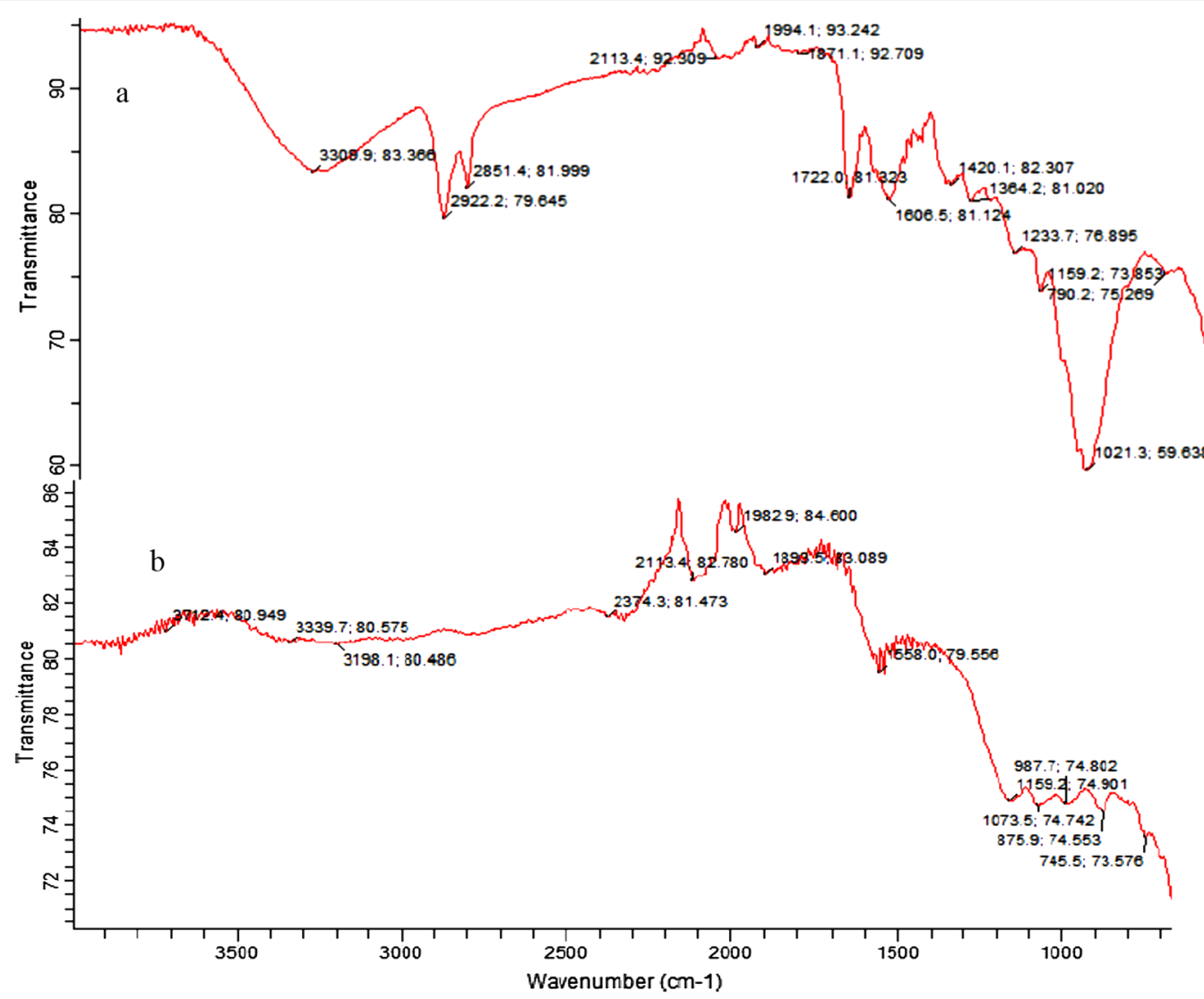

Fig. 3 FTIR Spectra of a raw Dialium guineense pods and $\mathbf{b}$ Dialium guineense pods active carbon
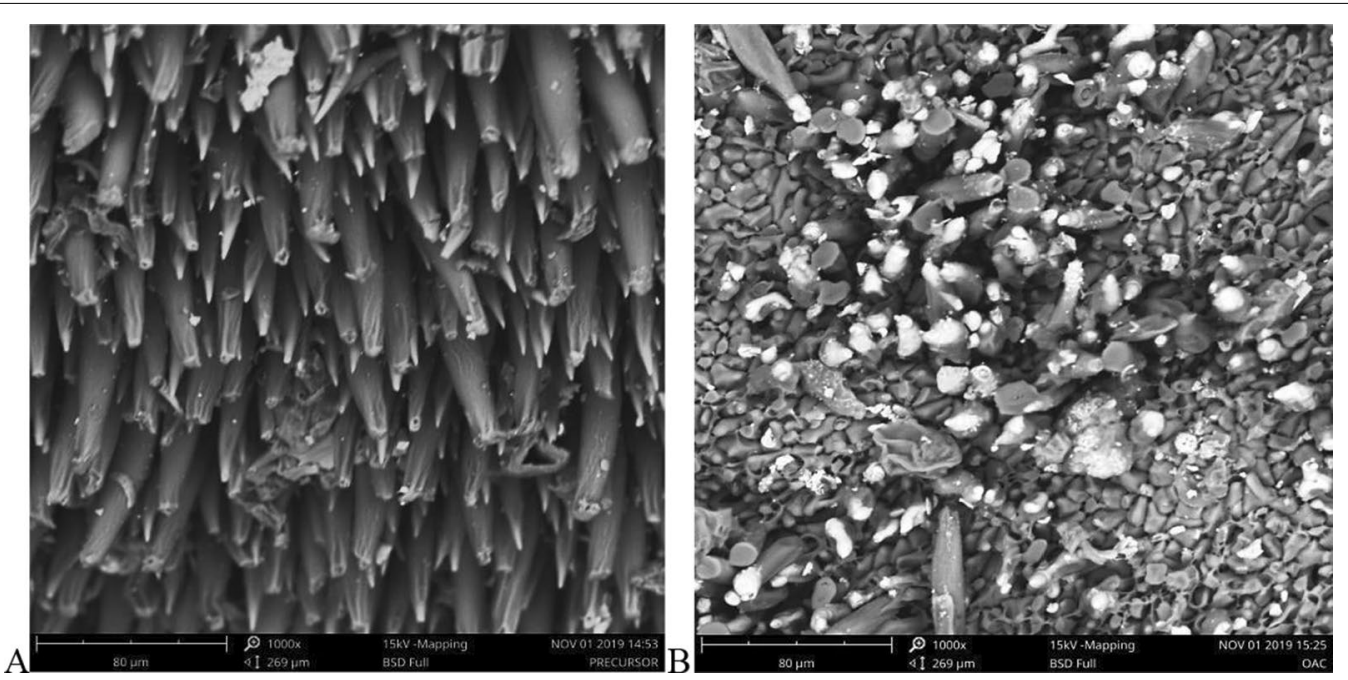

Fig. 4 SEM micrographs of a raw Dialium guineense pods and $\mathbf{b}$ Dialium guineense pods active carbon 
a negative effect on the response $\left(Y_{1}\right)$, implying that the activated carbon yield decreased as these terms increased. According to the signs of their coefficients, all linear terms $A$ (concentration of activating agent), $B$ (activation time), and $C$ (activating agent) in Eq. (3) had a positive effect on the adsorption capacity of DGPAC for MB, with A having the greatest influence as evidenced by the magnitude of its coefficient. This implies that an increase in the concentration of the activating agent results in a corresponding increase in adsorption capacity.

The ANOVA results for DGP active carbon yield reveals that of all three linear factors, only concentration of activating agent $(A)$ and activated time $(B)$ had a statistically significant effect on the activated carbon yield while the interaction term of $A B$ was also significant. The overall significance of the model was high as indicated by its low $p$-value thus verifying its sufficiency. The $R^{2}$ value obtained from the model was relatively high indicating that there was a good correlation between the experimental and the theoretical values from the model. The predicted $R$-squared, $R^{2}$ (pred) value, which is a measure of how well the model predicts a response value was in reasonable agreement with the adjusted $R$-squared $R^{2}$ (adj). The $R^{2}$ (adj) and $R^{2}$ (pred) should be within approximately $20 \%$ of each other to be in reasonable agreement (Das and Mishra 2017). This agreement confirms the good adequacy and predictability of the model.

From the ANOVA results for the adsorption capacity of DGPAC for MB, it was observed that activation time $(B)$ had no statistically significant effect on the adsorption capacity with a $p$-value much greater than 0.05 . The term was however retained in the model being a hierarchical one. Similar results have been reported by Jasper et al. (2019). The magnitude of the coefficient $A$ (concentration of activating agent) as seen from the model equation, and its $F$ value were highest indicating that the concentration of the activating agent had the most significant effect on the dye adsorption capacity. A high $R^{2}$ value was obtained indicating a good association between the measured and the predicted adsorption capacity values. The correlation between the $R^{2}$ (pred) and $R^{2}$ (adj) was also agreeable.

\section{Interpretation of Pareto and normal plots}

For activated carbon yield, at a 95\% confidence level and three degrees of freedom, three values higher than reference value $2.477(P=0.05)$ were located at the right of the dashed line and were significant in the order of $A>B>$ interaction term $A B$. The normal plot of this response showed that $A$ and $B$ had a negative influence on the response, while the interaction term had a positive influence on the response with $A$ exerting the most statistically significant effect.
For the adsorption capacity of DGPAC for MB, at a 95\% confidence level and seven degrees of freedom, five values higher than the reference value were located at the right of the reference line with the linear term $A$ (concentration of activating agent) being the most significant, followed by the type of activating agent $(C)$. All the two-way interaction terms were significant in the order $A C>A B>B C$. The normal plot of adsorption capacity of DGPAC for MB shows that linear terms, $A$ and $C$ and interaction terms $A C$ and $B C$ had a significant and positive effect on the response, while the interaction term $A B$ had a negative influence on the response. $A$ (concentration of the activating agent) had the most significant positive influence on the response.

The statistical results obtained revealed that $A$ (concentration of activating agent) and $B$ (activation time) have a significant positive influence on the yield of the active carbon while the concentration and type of activation agent $(A$ and $C$ ) had the most influence on the adsorption capacity of the DGPAC. Overall, the concentration of the activating agent had the most statistically significant influence on both responses thus being the factor that influenced the preparation process the most. Having identified these key factors, the settings that yield the best performance of the preparation process are further explored.

\section{Optimization}

The experimental values obtained at the software generated optimum settings for the preparation of the activated carbon were observed to be in close agreement with the values theoretically calculated from the models as they fell within the predicted intervals generated by the software. Thus, the FFD proved to be effective in optimizing the experimental conditions.

\section{FTIR and SEM analysis of DGP and DGPAC}

The FTIR spectra obtained for the optimized activated carbon showed fewer strong peaks than its precursor and this could be attributed to the cracking of some bonds as a result of heating. The broad $\mathrm{OH}$ stretching peak present in the spectrum of the precursor, decreased in intensity compared to DGPAC indicating the loss of hydroxyls (García-Saldaña et al. 2016). The attenuation of these peaks in the activated carbon shows the removal of aliphatics and aromatics eliminated as volatile substrates from its precursor (Yagmur et al. 2008).

A distinct difference in the surface morphology of the raw Dialium guineense seed pods and the optimal Dialium guineense seed pods activated carbon is indicative 
of a transformation of the precursor into a new product resulting from the activation and carbonization process. The appearance of several distinct pores in the SEM micrograph of the activated carbon when compared to the precursor is also an indication of a successful conversion of the precursor into activated carbon.

\section{Conclusions}

A full factorial experimental design was employed to study the effects of three active carbon preparation variables, namely, the activation agent concentration, activation time, and type of activating agent, on the Dialium guineense active carbon yield and adsorption capacity for MB. Regression model equations were developed to correlate the preparation variables to the two responses. From the analysis of the data derived from the models, the concentration of the activating agent was found to have the most significant effect on the active carbon yield and the adsorption capacity for MB. Upon optimization of the preparation process, the experimental values obtained for the active carbon yield and $\mathrm{MB}$ adsorption capacity were found to be agreeable with the values predicted by the models.

The FFD proved to be useful in identifying factors that significantly influence the preparation of Dialium guineense seed pods' active carbon.

\section{Abbreviations \\ ${ }^{\circ} \mathrm{C}$ : Degrees centigrade; A: Concentration of activating agent; Adj MS: Adjusted mean of squares; Adj SS: Adjusted sum of squares; ANOVA: Analysis of vari- ance; B: Activation time; C: Type of activating agent; DF: Degrees of freedom; DGP: Dialium guineense Seed Pods; DGPAC: Dialium guineense Seed Pods Active Carbon; FFD: Full factorial design; FTIR: Fourier transform infrared; MB: Methylene blue; OFAT: One factor at a time; SEM: Scanning electron micros- copy; $Y_{1}$ : Active carbon yield; $Y_{2}$ : Methylene blue adsorption capacity.}

\section{Acknowledgements}

We are thankful to Odo Peter who assisted with the laboratory work and to the Department of Applied Chemistry, Kaduna Polytechnic, and the Multi-User Science Research Laboratory of the Ahmadu Bello University, Zaria, for providing the infrastructure and instrument facility to carry out the research work.

\section{Authors' contributions}

EEJ and JCE conceptualized and designed the research. EEJ and YMB carried out the experiments. All the authors participated in analyzing the results and drafting the manuscript. All authors read and approved the final manuscript.

\section{Funding}

This research did not receive any specific grant from funding agencies in the public, commercial, or not-for-profit sectors,

\section{Availability of data and materials}

All data generated or analyzed during this study are included in this published article.

\section{Declarations}

Ethics approval and consent to participate

Not applicable.
Consent for publication

Not applicable.

\section{Competing interests}

The authors declare that they have no competing interests.

\section{Author details}

'Department of Applied Chemistry, Kaduna Polytechnic, Kaduna, Kaduna State, Nigeria. ${ }^{2}$ Chemical Science Technology Unit, Department of Science Laboratory Technology, Federal University of Lafia, Lafia, Nasarawa State, Nigeria.

Received: 26 April 2021 Accepted: 27 September 2021

Published online: 09 October 2021

\section{References}

Albert M, Lessin MS, Gilchrist BF (2003) Methylene blue: dangerous dye for neonates. J Pediatr Surg 38:1244-1245

Bae W, Kim J, Chung J (2014) Production of granular activated carbon from food-processing wastes (walnut shells and jujube seeds) and its adsorptive properties. J Air Waste Manag Assoc 64(8):879-886

Bingol D, Tekin N, Alkan M (2010) Brilliant yellow dye adsorption onto sepiolite using a full factorial design. Appl Clay Sci 50(3):315-321

Campos NF, Guedes GAJC, Oliveira LPS, Gama BMV, Sales DCS, Rodríguez-Díaz JM, Barbosa CMBM, Duarte MMB (2020) Competitive adsorption between $\mathrm{Cu}^{2+}$ and $\mathrm{Ni}^{2+}$ on corn cob activated carbon and the difference of thermal effects on mono and bicomponent systems. J Environ Chem Eng 8(5):104232

Das S, Mishra S (2017) Box-Behnken statistical design to optimize preparation of activated carbon from Limonia Acidissima shell with desirability approach. J Environ Chem Eng 5:588-600

Derringer G, Suich R (1980) Simultaneous optimization of several response variables.J Qual Technol 12:214-219

El-Hendawy AA (2006) Variation in the FTIR spectra of a biomass under impregnation, carbonization and oxidation conditions. J Anal Appl Pyrolysis 75(2):159-166

El Haddad M, Slimani R, Mamouni R, El Antri S, Lazar S (2013) Removal of two textile dyes from aqueous solutions onto calcined bones. J Assoc Arab Univ Basic Appl Sci 14:51-55

García-Saldaña JS, Campas-Baypoli ON, López-Cervante JS, Sánchez-Machado DI, Cantú-Soto EU, Rodríguez-Ramíre R (2016) Microencapsulation of sulforaphane from broccoli seed extracts by gelatin/gum arabic and gelatin/ pectin complexes. Food Chem 20:194-100

Geyikci F, Büyükgüngör H (2013) factorial experimental design for adsorption silver ions from water onto montmorillonite. Acta Geodyn Geom 10:363-370. https://doi.org/10.13168/agg.2013.0035

Gillman PK (2011) CNS toxicity involving methylene blue: the exemplar for understanding and predicting drug interactions that precipitate serotonin toxicity. J Psychopharmacol 25:429-436

Gouamid M, Mohammed RO, Massioud B (2013) Adsorption, equilibrium, kinetics and thermodynamics of methylene blue from aqueous solutions using date palm leaves. Energy Procedia 36:898-907

Jasper EE, Ajibola VO, Agbaji EB, Onwuka JC (2019) Optimization of the preparation of Millettia thonningii seed pods activated carbon for use in the remediation of dye-contaminated aqueous solutions. SN Appl Sci 1:1351-1363. https://doi.org/10.1007/s42452-019-1361-z

Kalavathy MH, Regupathi I, Pillai MG, Miranda LR (2009) Modeling, analysis and optimization of adsorption parameters for $\mathrm{H}_{3} \mathrm{PO}_{4}$ activated rubber wood sawdust using response surface methodology (RSM). Colloids Surf B 70:35-45

Kumari G, Soni B, Karmee SK (2020) Synthesis of activated carbon from groundnut shell via chemical activation. J Inst Eng India Ser E. https://doi.org/10. 1007/s40034-020-00176-z

Kyzas GZ, Lazaridis NK, Kostoglou M (2013) On the Simultaneous adsorption of a reactive dye and hexavalent chromium from aqueous solutions onto grafted chitosan. J Colloid Interface Sci 407:432-441

Menya E, Olupot PW, Storz H, Lubwama M, Kiros Y (2020) Synthesis and evaluation of activated carbon from rice husks for removal of humic acid from water. Biomass Conv Bioref. https://doi.org/10.1007/s13399-020-01158-2 
Montgomery DC (2013) Design and analysis of experiments, 8th edn. Wiley, Hoboken

Reddy KSK, Al Shoaibi A, Srinivasakannan C (2015) Preparation of porous carbon from date palm seeds and process optimization. Int J Environ Sci Technol 12:959-966

Wang K, Zhang Z, Sun Q et al (2020) Durian shell-derived N, O, P-doped activated porous carbon materials and their electrochemical performance in super capacitor. J Mater Sci. https://doi.org/10.1007/s10853-020-04740-1

Yagmur E, Ozma M, Akts Z (2008) A novel method for production of activated carbon from waste tyre by chemical activation with microwave energy. Fuel 87:327-3285
Yagub MT, Sen TK, Afroze S, Ang HM (2014) Dye and its removal from aqueous solution by adsorption: a review. Adv Colloid Interface Sci 209:172-184

\section{Publisher's Note}

Springer Nature remains neutral with regard to jurisdictional claims in published maps and institutional affiliations.

\section{Submit your manuscript to a SpringerOpen ${ }^{\circ}$ journal and benefit from:}

- Convenient online submission

- Rigorous peer review

- Open access: articles freely available online

- High visibility within the field

- Retaining the copyright to your article

Submit your next manuscript at $\boldsymbol{\nabla}$ springeropen.com 